\title{
Cardiotoxicity and Serotonin Syndrome Complicating a Milnacipran Overdose
}

\author{
Michael Levine • Carrie A. Truitt • Ayrn D. O'Connor
}

Published online: 7 July 2011

(C) American College of Medical Toxicology 2011

\begin{abstract}
Milnacipran is a selective serotonin and norepinephrine reuptake inhibitor, recently approved for use in the USA for treatment of fibromyalgia. This case report describes a 59-year-old woman who ingested 3,000 mg of milnacipran in a suicide attempt. Following the ingestion, she became obtunded and developed autonomic instability. She required mechanical ventilation, treatment for hypertension, and then ultimately vasopressor support for refractory hypotension. In addition, she developed a transient, acute cardiac dysfunction with global hypokinesis and an ejection fraction of $30 \%$. Resolution of the cardiac dysfunction was documented on repeat echocardiogram 2 days after the initial study. This was confirmed by cardiac catheterization performed 4 days after the acute ingestion in which coronary arteriogram was normal and left ventricular ejection fraction was $70 \%$. Acute overdose was confirmed by quantification of plasma milnacipran concentration of $8,400 \mathrm{ng} / \mathrm{mL}$ obtained $5 \mathrm{~h}$ post-ingestion. To our knowledge, this represents the first case of cardiac toxicity complicating a milnacipran overdose in the medical literature.
\end{abstract}

Keywords Milnacipran · Overdose · Cardiomyopathy · Fibromyalgia $\cdot$ Antidepressant $\cdot$ Serotonin syndrome

\section{Introduction}

Milnacipran is a selective serotonin and norepinephrine reuptake inhibitor (SNRI). It has been widely used as an

M. Levine $(\triangle) \cdot$ C. A. Truitt $\cdot$ A. D. O'Connor

Department of Medical Toxicology,

Banner Good Samaritan Medical Center,

Phoenix, AZ, USA

e-mail: Michael.Levine@bannerhealth.com antidepressant in Europe; however, experience in the USA is limited as it has only recently been Food and Drug Administration (FDA) approved for treatment of fibromyalgia. It is not yet approved for use as an antidepressant [1]. The typical dosing begins at $12.5 \mathrm{mg}$ daily, with dosage escalation to $25 \mathrm{mg}$ daily after several days. While acute overdoses of milnacipran have been described [2], reports remain rare and not well described in the literature. This case describes a 59-year-old woman with an acute stress cardiomyopathy following an acute milnacipran overdose.

\section{Case Report}

A 59-year-old, $70.5 \mathrm{~kg}$ woman called emergency medical services (EMS) $30 \mathrm{~min}$ following an intentional overdose of $3,000 \mathrm{mg}(42.6 \mathrm{mg} / \mathrm{kg})$ of milnacipran. The patient has a longstanding history of depression and had nine prior suicide attempts. During one such attempt, approximately 13 months prior, she had an echocardiogram that revealed a normal leftventricular ejection fraction (LVEF) of $65 \%$ without any regional wall motion abnormalities. In addition, her past medical history included hypertension, hypothyroidism, attention deficit hyperactivity disorder, and fibromyalgia. Her prescribed medications include methylphenidate, paroxetine, carisoprodol, milnacipran, methadone, and levothyroxine; however, she was noncompliant with this regimen.

EMS found the patient awake and alert with a blood pressure of 114/76 $\mathrm{mmHg}$ with a heart rate of 73 beats per minute (bpm). Paroxetine and diazepam bottles were found in the house although she denied ingesting any of these medications, and subsequent pill counts revealed only the milnacipran was missing. During transport to the emergency department (ED), the patient received $50 \mathrm{~g}$ of activated charcoal by EMS. Shortly thereafter, her mental status 
declined, and approximately $1 \mathrm{~h}$ post-ingestion, she became hypotensive with a blood pressure of $70 / 50 \mathrm{mmHg}$ with a heart rate of $60 \mathrm{bpm}$. In the emergency department, exam revealed an obtunded, diaphoretic patient with no gag reflex, and shallow respirations. Consequently, she was intubated with etomidate and succinylcholine. A norepinephrine infusion was initiated at $5 \mu \mathrm{g} / \mathrm{min}$ due to persistent hypotension.

Evaluation at the outside ED included an initial 12-lead electrocardiogram which revealed sinus bradycardia with a heart rate of $58 \mathrm{bpm}$. The QRS was $104 \mathrm{~ms}$, with a corrected QT interval of $488 \mathrm{~ms}$. Acetaminophen, salicylate, and ethanol levels were nondetectable, and a urine drug screen for drugs of abuse was positive for tetrahydrocannabinol and benzodiazepines. Her electrolytes and liver functions were normal. A post-intubation arterial blood gas revealed a pH of 7.31 with a pCO2 of $42 \mathrm{mmHg}$. She was transferred to a tertiary care medical center for admission to the toxicology service. During the transport, she received a single dose of $10 \mathrm{mg}$ vecuronium, and norepinephrine was titrated off.

She arrived with a blood pressure of $133 / 74 \mathrm{mmHg}$ and a heart rate of $67 \mathrm{bpm}$, chemically paralyzed, on versed and fentanyl infusions. An arterial line was placed for invasive hemodynamic monitoring. Approximately $6 \mathrm{~h}$ postingestion ( $1 \mathrm{~h}$ after arrival in the intensive care unit), the patient developed atrial fibrillation with a ventricular rate of approximately $130 \mathrm{bpm}$. The corresponding blood pressure was $249 / 145 \mathrm{mmHg}$. The patient received $20 \mathrm{mg}$ of intravenous diltiazem over $5 \mathrm{~min}$ with subsequent improvement in the heart rate to approximately $100 \mathrm{bpm}$. Approximately $15 \mathrm{~min}$ later, the blood pressure decreased to $66 / 54$. The patient received 21 of intravenous crystalloid, $10 \mathrm{ml}$ of $10 \%$ calcium gluconate, and $2 \mathrm{~g}$ of magnesium sulfate without improvement in the blood pressure. Norepinephrine infusion was initiated for persistent hypotension. Initial electrocardiography (ECG) revealed a sinus arrhythmia with an average rate of $130 \mathrm{bpm}$ and QRS of $94 \mathrm{~ms}$ and upright T waves. Subsequent ECGs revealed T wave flattening/inversion throughout the precordium these changes are summarized in Fig. 1. A stat bedside echocardiogram while on $10 \mu \mathrm{g} / \mathrm{min}$ of norepinephrine revealed an LVEF of $30-35 \%$ with moderate to severe diastolic dysfunction. Over the next hour, the norepinephrine was titrated to $20 \mu \mathrm{g} / \mathrm{min}$ and she was started on dobutamine at $10 \mu \mathrm{g} / \mathrm{kg} / \mathrm{min}$, which was titrated to a satisfactory mean arterial pressure. The patient required norepinephrine for an additional $38 \mathrm{~h}$ and dobutamine for $66 \mathrm{~h}$. The patient's troponin peaked at $0.45 \mathrm{ng} / \mathrm{mL}$.

The patient developed a fever of $38.9^{\circ} \mathrm{C} 2 \mathrm{~h}$ after arrival to the intensive care unit. Exam off sedation was significant for tremor of bilateral upper extremities, no clonus or rigidity was noted and patient gradually began to follow commands. Additional diagnostic evaluation included normal thyroid studies. A drug of abuse screen (immunoassay) revealed the presence of tetrahydrocannabinol and benzodiazepines. Comprehensive urine drug testing via gas chromatography/mass spectrometry (GC-MS) revealed the presence of paroxetine, nicotine, and milnacipran. No sympathomimetic agents including amphetamine or methylphenidate were detected. Of note, the area under the curve for paroxetine on the GC-MS was small, approximating the level of the internal standard. Five hours post-ingestion, a plasma milnacipran concentration performed by high performance liquid chromatography/tandem mass spectrometry was $8,400 \mathrm{ng} / \mathrm{mL}$ (mean maximal plasma concentration with therapeutic dosing $150 \mathrm{ng} / \mathrm{mL}$ [3].).

Two days post-ingestion, a repeat echocardiogram was performed and revealed resolution of the cardiac dysfunction with an LVEF of $60-65 \%$ without any regional wall motion abnormalities. Subsequent cardiac catheterization performed 4 days post-ingestion revealed normal coronary arteriogram, no regional wall motion abnormality, and an ejection fraction (EF) of $>70 \%$. Also of note, $\mathrm{T}$ wave inversion seen on ECG had resolved. The patient made a full recovery and ultimately was discharged to in-patient psychiatry.

\section{Discussion}

Milnacipran (1-phenyl-1-diethyl-aminocarbonyl-2-aminomethyl-cyclopropane ( $Z$ ) hydrochloride; Fig. 2) is a selective SNRI, developed and used as an antidepressant in Europe [4]. However, in the USA, it only recently gained FDA approval for use as a treatment for fibromyalgia [1]. At therapeutic dosing, there is no activity at the cholinergic, dopaminergic D2, adrenergic, muscarinic, or histaminergic H1 receptors [2, 4-6]. Based on its receptor sensitivity, neither sedation nor anticholinergic properties would be expected [7]. Following therapeutic administration of milnacipran, peak plasma concentrations occur between 0.5 and $4 \mathrm{~h}$ with a half-life of $8-10 \mathrm{~h} \mathrm{[3]}$. The volume of distribution is large $(5.3 \mathrm{~L} / \mathrm{kg})$ with little protein binding $(13 \%)[3,6]$. Following absorption, $50-60 \%$ of the drug is eliminated in the urine with $20 \%$ being excreted as a glucuronide conjugate. A small amount is excreted as $N$ dealkylated minlacipran with the remainder excreted as other metabolites [6]. Milnacipran does not undergo metabolism via the P450 system and none of the metabolites are pharmacologically active $[3,6]$.

Adverse effects during therapeutic administration are mild and primarily limited to gastrointestinal complaints and headache. Vertigo, increased sweating, anxiety, and dysuria, while relatively uncommon, did occur more than twice as often as placebo [8]. In therapeutic dosing, milnacipran is not associated with any changes on the electrocardiogram [2] or significant cardiovascular compli- 


\section{a}

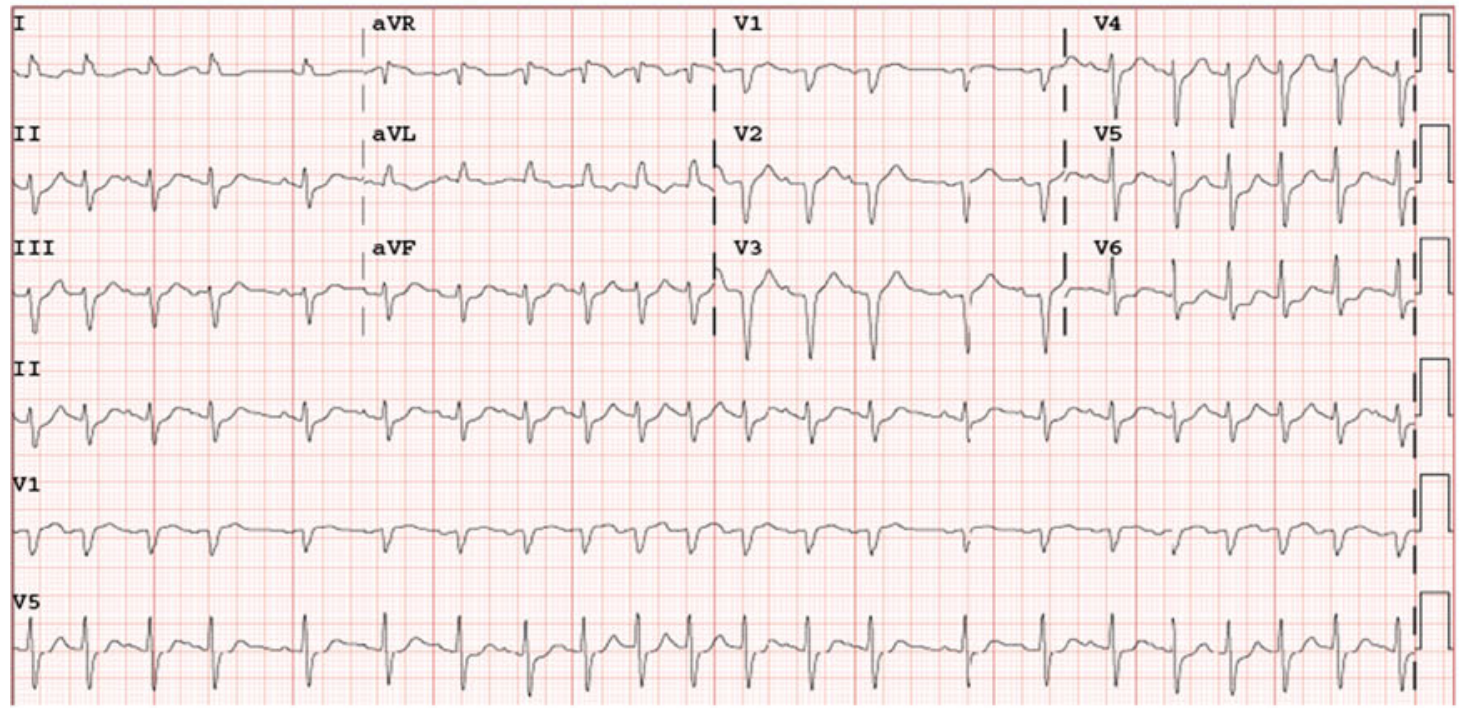

b

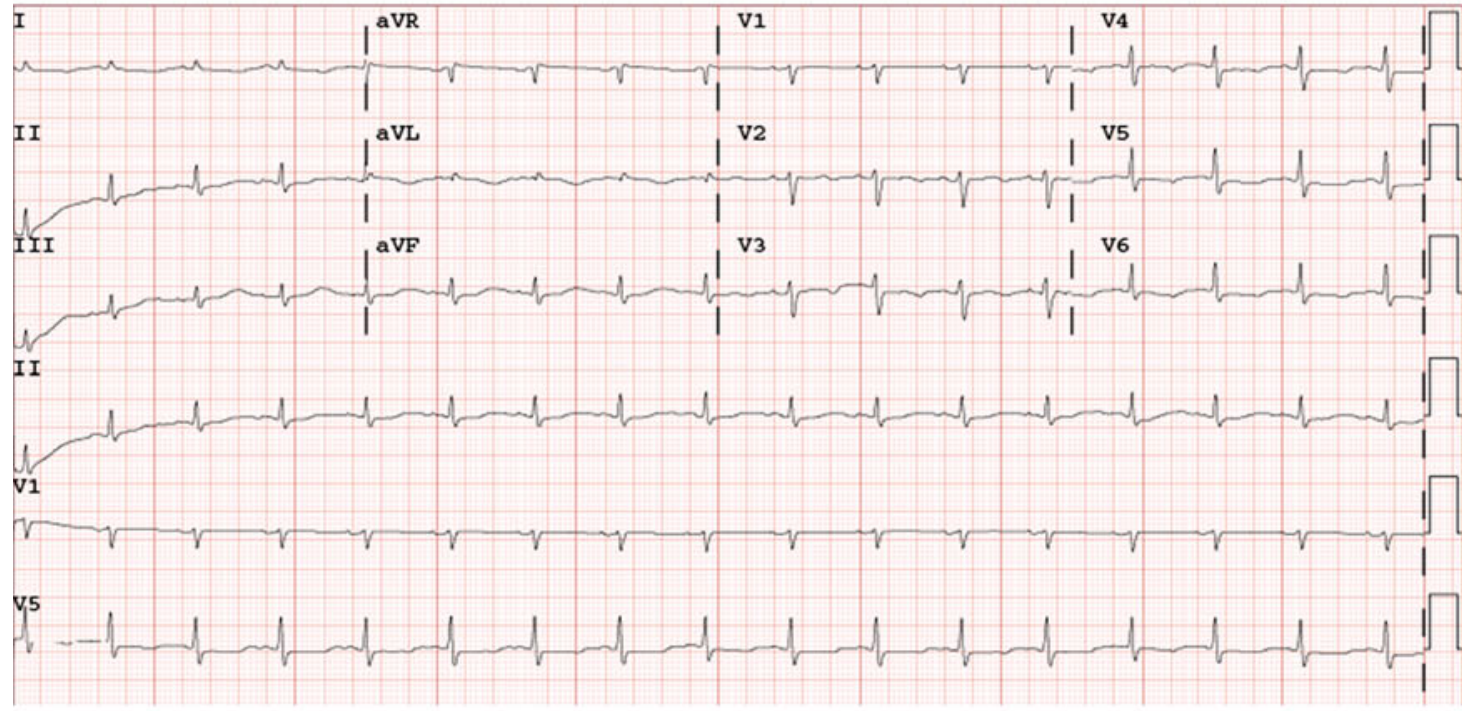

c

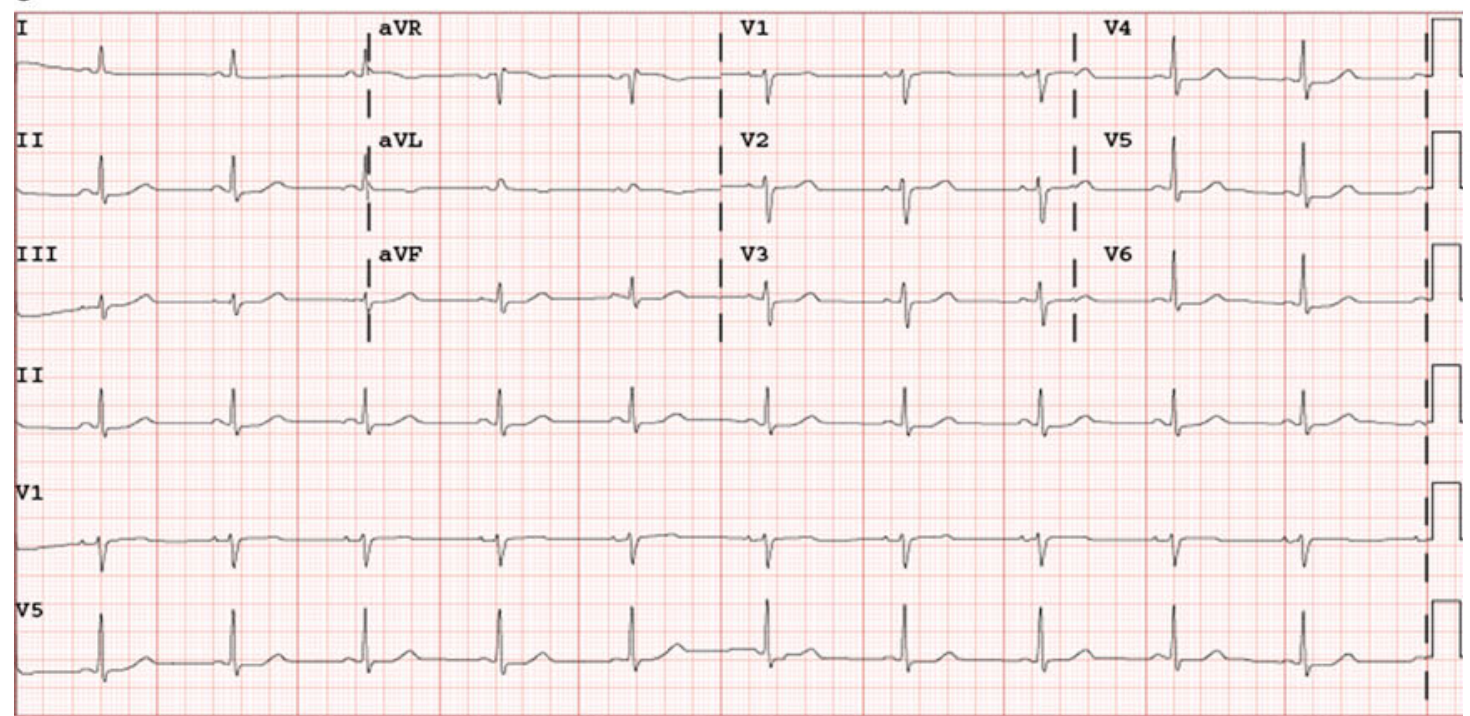


4Fig. 1 a Admission ECG. b ECG 1 day post-ingestion, demonstrating $\mathrm{T}$ wave flattening and inversion. c ECG 9 days post-ingestion, demonstrating resolution of ECG changes without Q waves

cations, including any dysrhythmias [9]. Drug-associated parkinsonism has rarely been reported during therapy with milnacipran [10]. During efficacy studies, 15 overdoses occurred either alone or in combination with other agents. The maximal dose ingested was 2,600 mg. An additional overdose involving 5,600 $\mathrm{mg}$ has been described in France but full details are not accessible. Fanton et al. have described a fatality in a 42-year-old female who was found dead in the back of a car. While numerous agents were suspected as part of her ingestion, only milnacipran concentrations were significantly elevated [11].

This case describes a 59-year-old woman who developed coma, respiratory failure, autonomic instability, tremor, and an acute cardiac dysfunction following milnacipran ingestion. This patient developed hypotension requiring norepinephrine infusion followed later by hypertension and tachycardia before again becoming hypotensive. The patient had been off norepinephrine for more than $1 \mathrm{~h}$ before the hypertension, tachycardia, and atrial fibrillation developed. Milnacipran does inhibit the reuptake of norepinephrine. Thus, it is possible that the concurrent use of milnacipran with norepinephrine can contribute to hypertensive emergencies and dysrhythmias [1]. While this drug interaction is certainly well documented, given the elimination kinetics of norepinephrine, we would have expected the increased sympathomimetic effects to have occurred during or immediately after the administration of norepinephrine. Thus, given that more than $1 \mathrm{~h}$ lapsed between the discontinuation of the norepinephrine infusion and the development of hypertension, tachycardia, and atrial dysrhythmia, we feel it is unlikely that an interaction between exogenous norepinephrine and milnacipran was the cause of the hyperadrenergic symptoms. Given the presence of CNS depression, autonomic instability, fever, and tremor the diagnosis of serotonin syndrome was raised. Clearly, this is a clinical diagnosis and difficult to confirm. The patient was taking an selective serotonin reuptake inhibitor therapeutically (paroxetine) and acutely overdosed on an SNRI (milnacipran); consequently, increased serotonergic tone would be expected. This

Fig. 2 Structure of milnacipran

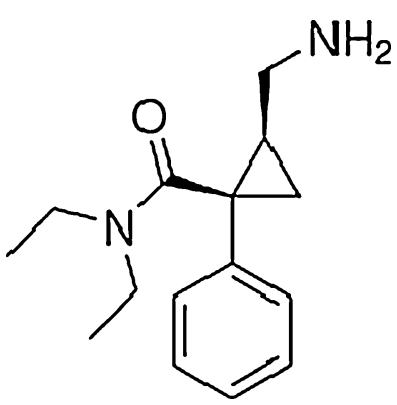

interaction coupled with the clinical presentation suggests serotonin syndrome. While therapeutic levels of paroxetine were not obtained, the patient reported only ingesting milnacipran and pill counts supported this history. Furthermore, the area under the curve on the comprehensive urine drug screen was consistent with therapeutic dosing. Consequently, the evidence supports her statement that the overdose was exclusively on milnacipran and the paroxetine was being taken therapeutically. Yacoub et al. describe a patient who was chronically taking paroxetine (25 mg daily) and was started on milnacipran (50 mg twice daily) 8 days prior to his presentation in which he was confused, lethargic, dysarthric, hyperreflexic, hypertensive, tachycardic, and hyperthermic. Serotonin syndrome was diagnosed after thorough work-up failed to reveal an infectious cause or any other medical etiology [12].

The development of significant hypertension and rapid atrial fibrillation necessitated treatment with diltiazem, shortly thereafter the patient became hypotensive requiring vasopressor support. The prolonged duration of the patient's hypotension could not be attributed to an adverse effect of a single intravenous dose of diltiazem $(20 \mathrm{mg})$. Echocardiography confirmed the development of an acute cardiac dysfunction, with moderate to severe diastolic dysfunction and an LVEF of $30-35 \%$. Experimentally, high dose $(10 \mathrm{mg} / \mathrm{kg})$ of intravenous milnacipran has been shown to have negative inotropic, chronotropic, and dromotropic effects suggesting a possible direct cardiotoxicity of the drug [13]. However, this patient had hypertension and tachycardia, followed by hypotension and bradycardia. These later vital sign abnormalities, along with the reversible, global effects on the echocardiogram, suggest the development of stress-induced cardiac dysfunction. Stress-induced cardiomyopathy by definition is completely reversible acute LV dysfunction. Clinical characteristics of stress-induced cardiomyopathy include anterior subtle ST elevations, deep T wave inversions on ECG, borderline troponins with peak usually $<5 \mathrm{ng} / \mathrm{mL}$, abnormalities on echocardiogram, and normal coronaries on angiogram [14]. Findings on ECHO include akinesis of the mid and distal segments of LV with hypercontractile base in $60-80 \%$ of patients, apical sparing is seen in $10 \%$ and the remainder manifests global hypokinesis or nonspecific regional wall motion abnomalities [14]. The pathogenesis of stress cardiomyopathy is thought to be due to catecholamine excess which mediates the acute cardiac dysfunction which can be seen following acute physiologic or mental stress. In addition to the presumed emotional distress leading to the patient's suicide attempt, the acute milnacipran overdose may have resulted in a catecholamine excess, with subsequent development of stress cardiomyopathy. The global dysfunction on the echocardiogram had completely resolved on repeat echocardiography performed 2 days later. A cardiac catheterization performed 4 days post-ingestion revealing 
normal coronary arteries and LVEF of $>70 \%$ supports the diagnosis of a stress-induced cardiomyopathy. Christoph et al. reported a case of tako tsubo cardiomyopathy following venlafaxine overdose [15]. Like the patient described in their report our patient developed inverted $\mathrm{T}$ waves in the precordial leads and slightly elevated troponin; however, our patient lacked the classic apical akinesis with basal hypercontractility typical of apical ballooning. Nonetheless, global hypokinesis with severely decreased EF in the face of normal coronary arteries that rapidly resolved supports the diagnosis of stress cardiomyopathy. This coupled with quantitative milnacipran levels $(8,400 \mathrm{ng} / \mathrm{mL})$, comprehensive drug testing excluding other co-ingestants and corroborating history of acute overdose supports milnacipran as a contributing agent.

\section{Conclusion}

Overdoses of milnacipran resulting in significant toxicity are exceedingly rare. We believe this represents the first case of acute cardiac dysfunction associated with acute milnacipran overdose.

Conflict of Interest There are no financial, litigational, or other conflicts of interest involved in the preparation of this manuscript.

\section{References}

1. Forest Laboratories, Inc (2009) Product information: Savella oral tablets, milnacipran $\mathrm{HCl}$ oral tablets. Forest Laboratories, Inc., NY
2. Montgomery SA, Prost JF, Solles A et al (1996) Efficacy and tolerability of milnacipran: an overview. Int Clin Psychopharmacol 11(suppl 4):47-51

3. Puozzo C, Leonard BE (1996) Pharmacokinetics of milnacipran in comparison with other antidepressants. Int Clin Psychopharmacol 11(suppl 4):15-27

4. Briley M, Prost JF, Moret C (1996) Preclinical pharmacology of milnacipran. Int Clin Psychopharmacol 11(suppl 4):10-14

5. Assie MB, Charveron M, Palmier C et al (1992) Effects of prolonged administration of milnacipran, a new antidepressant, on receptors and monoamine uptake in the brain of the rat. Neuropharmacology 31:149-155

6. Boyer P, Briley M (1998) Milnacipran, a new specific serotonin and noradrenaline reuptake inhibitor. Drug Today (Barc) 34:709-720

7. Moret C, Charveron M, Finberg JP et al (1985) Biochemical profile of midalcipran (F 2207), 1-phenyl-1-diethyl-aminocarbonyl-2-aminomethyl-cyclopropane ( $\mathrm{Z}$ ) hydrochloride, a potential fourth generation antidepressant drug. Neuropharmacology 24:1211-1219

8. Lopez-Ibor J, Guelfi JD, Pletan Y et al (1996) Milnacipran and selective serotonin reuptake inhibitors in major depression. Int Clin Psychopharmacol 11(suppl 4):41-46

9. Ikawa M, Yamada K (2010) Effectiveness of milnacipran for a patient with pain disorder and long QT syndrome. Clin Neuropharmacol 33:163-164

10. Muraoka T, Oku E, Sugataka K et al (2008) A case of severe parkinsonism associated with short-term treatment with milnacipran. Clin Neuropharmacol 31:299-300

11. Fanton L, Bevalot F, Grait $\mathrm{H}$ et al (2008) Fatal intoxication with milnacipran. J Forensic Leg Med 15:388-390

12. Yacoub HA, Johnson WG, Souayah N (2010) Serotonin syndrome after administration of milnacipran for fibromyalgia. Neurology 74:699-700

13. Mitsumori Y, Nakamura Y, Hoshiai K et al (2010) In vivo canine model comparison of cardiovascular effects of antidepressants milnacipran and imipramine. Cardiovasc Toxicol 10:275-282

14. Chockalingam A, Mehra A, Dorairajan S et al (2010) Acute left ventricular dysfuction in the critically ill. Chest 138(1):198-207

15. Christoph M, Ebner B, Stolte D et al (2010) Broken heart syndrome: Tako Tsubo cardiomyopathy associated with an overdose of the serotonin-norepinephrine reuptake inhibitor venlafaxine. Eur Neuropsychomarmacol 20:594-597 